\title{
Observing thermal Schwinger pair production
}

\author{
Oliver Gould,,${ }^{1,2, *}$ Stuart Mangles,,${ }^{1,}$ Arttu Rajantie, ${ }^{1, \star}$ Steven Rose,,${ }^{1,}$ and Cheng Xie ${ }^{1, \|}$ \\ ${ }^{1}$ Department of Physics, Imperial College London, London SW7 2AZ, United Kingdom \\ ${ }^{2}$ Helsinki Institute of Physics, University of Helsinki, Helsinki FI-00014, Finland
}

(Received 8 January 2019; published 22 May 2019)

\begin{abstract}
We study the possibility of observing Schwinger pair production enhanced by a thermal bath of photons. We consider the full range of temperatures and electric field intensities from pure Schwinger production to pure thermal production, and identify the most promising and interesting regimes. In particular, we identify temperatures of $\sim 20 \mathrm{keV} / k_{B}$ and field intensities of $\sim 10^{23} \mathrm{~W} \mathrm{~cm}^{-2}$ where pair production would be observable. In this case, the thermal enhancement over the Schwinger rate is exponentially large and due to effects which are not visible at any finite order in the loop expansion. Pair production in this regime can thus be described as more nonperturbative than the usual Schwinger process, which appears at one loop. Unfortunately, such high temperatures appear to be out of reach of foreseeable technologies, though nonthermal photon distributions with comparable energy densities are possible. We suggest the possibility that similar nonperturbative enhancements may extend out of equilibrium and propose an experimental scheme to test this.
\end{abstract}

DOI: 10.1103/PhysRevA.99.052120

\section{INTRODUCTION}

Schwinger predicted long ago that a strong, constant electric field will create electron-positron pairs [1]. This has, however, never been experimentally observed as the electric field strengths required are several orders of magnitude larger than has ever been achieved in the laboratory $[2,3]$. The rate of pair production becomes large as the electric field strength approaches an appreciable fraction of the Schwinger critical field, $E_{c}=m_{e}^{2} c^{3} / e \hbar$, corresponding to an electric field intensity $I_{c}=\frac{1}{2} \epsilon_{0} c E_{c}^{2} \approx 2 \times 10^{29} \mathrm{~W} \mathrm{~cm}^{-2}$. It was also predicted long ago that one can create electron-positron pairs from a thermal bath of photons [4] via the two-photon Breit-Wheeler process, $\gamma \gamma \rightarrow e^{+} e^{-}$[5]. This too has never been observed due to the unattainability of the high temperatures required (although an experiment has been proposed that uses a quasithermal radiation field for one of the two photons [6]). In this case, the rate of pair production becomes large when $k_{B} T$ becomes an appreciable fraction of the rest mass energy of an electron and positron, $2 m_{e} c^{2} \sim 1 \mathrm{MeV}$.

One can, however, combine these two ingredients. Starting from an initial state containing a thermal bath of photons at a high temperature and adding a strong, constant electric field, one finds the rate of pair production is significantly faster than either the Schwinger process or the purely thermal process alone. The nature of the process depends on the relative magnitudes of the electric field strength and the temperature. At low temperatures, when $E / E_{c} \gg k_{B} T / 2 m_{e} c^{2}$, the process is essentially Schwinger pair production, in which virtual electron-positron pairs tunnel quantum mechanically

\footnotetext{
*oliver.gould@helsinki.fi

†stuart.mangles@imperial.ac.uk

¥a.rajantie@imperial.ac.uk

§s.rose@imperial.ac.uk

" cxie@live.com
}

through their energy barrier to existence. In the opposite limit, $E / E_{c} \ll k_{B} T / 2 m_{e} c^{2}$, at high temperatures, virtual electron-positron pairs are given sufficient energy from the thermal bath to transition over the barrier classically. At intermediate temperatures, the process can be described as thermally enhanced quantum tunneling, whereby the virtual electron-positron pairs tunnel from an excited state at nonzero separation.

Here we consider the viability of observing the thermal Schwinger process. If this were realized, it would be the first observation of semiclassical pair production in quantum electrodynamics (QED), a class of nonperturbative phenomena with applications in many branches of physics, including astrophysics, cosmology, heavy-ion collisions, and plasma physics (see, for example, [7]). It would also open up the controlled study of semiclassical pair production in general, a very basic process in quantum field theory and one that has proved elusive experimentally.

To put our work in context, we note that several other mechanisms have been proposed to lower the intensities required for Schwinger pair production, by including highfrequency fields [8-17] or the Coulomb fields of highly charged nuclei [18-21]. Further, it has been pointed out that once an initial seed pair has been produced, a cascade of pair production will follow for sufficiently strong field intensities [22-24]. This may dramatically amplify any signal of Schwinger pair production. On the experimental side of strong-field QED, there has been much progress on a variety of fronts [25-30], and the next generation of high-intensity lasers offers exciting possibilities to discover and investigate qualitatively new phenomena in QED [3,31].

In the regime we consider here, there is an additional significance, in that the formula for the rate of pair production is a rare example of an all-orders and all-loop result in QED $[32,33]$. Perturbative estimates of the rate of pair production are orders of magnitude slower, so they could be distinguished experimentally. Hence, one can probe QED beyond the 
perturbative loop expansion and could decide experimentally on the validity of conjectured all-order behaviors of QED [32-37]. Further, an experimental verification of the formulas would directly carry over to strongly coupled physics, and hence provide an important validation of the principle in the search for magnetic monopoles [38,39]. As such, an experimental search for the thermal Schwinger process is well motivated even independently of its connection to the pure Schwinger process.

\section{THEORETICAL RESULTS}

At zero temperature and for electric field strengths somewhat below $E_{c}$, the rate of electron-positron pair production per unit volume in a constant electric field is given by Schwinger's result [1],

$$
\Gamma_{\text {Schwinger }}(E) \approx \frac{(e E)^{2}}{4 \pi^{3} c \hbar^{2}} e^{-\frac{\pi m_{e}^{2} c^{3}}{e E \hbar}} .
$$

Loop corrections to this formula have been computed at two loops [36,40-42] (see also [43]) and have been resummed to all loops (within the quenched approximation) [35,36]. However, the loop corrections are small and only give an $O(1 \%)$ enhancement over Schwinger's one-loop result. At leading loop order, there are also corrections which are exponentially subdominant for small $E / E_{c}[1]$.

If one adds a thermal bath of photons, the rate is enhanced. Note that in this analysis, it is crucial that there are very few charged particles in the initial thermal state as their presence would Debye screen the electric field. The Debye screening length should be longer than the scales relevant for pair production (which we give in Sec. IV). Hence, we assume $k_{B} T \ll m_{e} c^{2}$.

Depending on the relative magnitudes of $E$ and $T$, one finds three different regimes. In the lowest-temperature regime, the energy of the thermal bath is less than the energy that an electron-positron pair would gain when accelerated by the electric field over their Compton wavelength; that is, for temperatures lower than

$$
T_{\mathrm{CW}}:=e E \hbar /\left(2 m_{e} c k_{B}\right) \text {. }
$$

In this regime, the thermal bath is negligible and electronpositron pairs are produced by quantum tunneling from virtuality in vacuum, to reality. Above $T_{\mathrm{CW}}$, the thermal bath excites virtual electron-positron pairs significantly above their ground state. Pair production then takes the form of quantum tunneling from an excited state. At higher temperatures still, virtual electron-positron pairs acquire sufficient energy from thermal fluctuations to go over the energy barrier classically. This process dominates over quantum tunneling for temperatures greater than

$$
T_{\mathrm{WS}}:=\left(4 e E^{3} \epsilon_{0} \hbar^{4} / \pi^{3} m_{e}^{2} k_{B}^{4}\right)^{1 / 4} .
$$

This temperature can be understood as that when the lowest thermal (Matsubara) frequency $2 \pi k_{B} T / \hbar$ is equal to the exponential decay rate of the unstable electron-positron transition state, $2 \sqrt{2}\left(e E^{3} \epsilon_{0} / m_{e}^{2}\right)^{1 / 4}$. $^{1}$

\footnotetext{
${ }^{1}$ The labels $C, W$, and $S$ follow the notation of Ref. [32]. They stand for circular, wavy, and straight (or sphaleron), respectively, and refer to the shape of the instanton describing the processes at low, intermediate, and high temperatures.
}

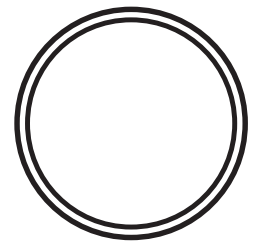

(a)

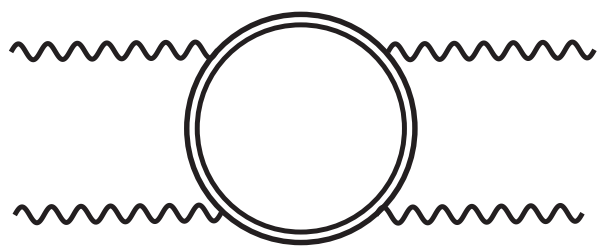

(b)

FIG. 1. Feynman diagrams which dominate the rate of thermal Schwinger pair production in the (a) low- and (b) intermediatetemperature regimes. Double lines denote the electron propagator including the effect of the external electric field to all orders and the wiggly lines denote photons from the thermal bath. In the hightemperature regime, the rate is not dominated by a single such Feynman diagram, but infinitely many diagrams contribute to the leading approximation to the rate.

Low temperatures, $T<T_{\mathrm{CW}}$. For very low temperatures, $T \ll T_{\mathrm{CW}}$, corrections to the Schwinger rate can be considered perturbatively. The dominant contribution to the rate in this regime is thus given by Fig. 1(a), just as at $T=0$. The leading thermal corrections arise at two loops and are [44]

$$
\Gamma_{C}=\Gamma_{\text {Schwinger }}\left[1+\frac{\pi^{4} e^{2}}{1440 \epsilon_{0} \hbar c}\left(\frac{T}{T_{\mathrm{CW}}}\right)^{4}+\cdots\right] .
$$

As $T$ increases towards $T_{\mathrm{CW}}$, higher-order perturbative corrections become important. The exponential enhancement due to these corrections has been computed at leading [45] and next-to-leading [32] order. The corrections to the exponent of the rate are small if $E \lesssim E_{c}$, except if one is very close to $T_{\mathrm{CW}}$ in which case even the exponent is unknown. Where one can trust the calculations, the thermal enhancement at low temperatures is less than $1 \%$.

Intermediate temperatures, $T_{\mathrm{CW}}<T<T_{\mathrm{WS}}$. At $T_{\mathrm{CW}}$, the rate goes through a sharp transition. In this intermediatetemperature range, the rate of pair production is significantly (exponentially) enhanced by the presence of the thermal bath. For intermediate temperatures, the exponent of the rate is given by [32,46-49]

$$
\begin{gathered}
\Gamma_{W}(E, T) \sim \exp \left\{-2 \frac{m_{e}^{2} c^{3}}{e E \hbar}\left[\arcsin \left(\frac{T_{\mathrm{CW}}}{T}\right)\right.\right. \\
\left.\left.+\frac{T_{\mathrm{CW}}}{T} \sqrt{1-\frac{T_{\mathrm{CW}}^{2}}{T^{2}}}\right]\right\},
\end{gathered}
$$

though there has been some dispute on this [50,51]. The precise formula, including the prefactor of the exponential, has recently been worked out in Ref. [52]. There it was also shown that the dominant contribution to the rate in this regime is given by Fig. 1(b). 
High temperatures, $T_{\mathrm{WS}}<T$. At $T_{\mathrm{WS}}$, there is again a sharp transition in the rate. In the high-temperature regime, the pair-production process can be seen as a thermal process enhanced by the presence of the electric field. Unlike the lower-temperature regimes, the rate is not dominated by the diagrams in Fig. 1; infinitely more such diagrams contribute to the leading approximation to the rate, leading to a significant nonperturbative enhancement. Some of the present authors have recently calculated the rate $[32,33]$. It is given by

$$
\Gamma_{S}(E, T) \approx \frac{4 k_{B} T_{\mathrm{WS}}\left(m_{e} k_{B} T\right)^{3 / 2} e^{-\frac{2 m_{e} e^{2}}{k_{B} T}+\frac{\sqrt{e^{3} E / \pi \epsilon_{0}}}{k_{B} T}}}{(4 \pi)^{3 / 2} \hbar^{4} \sin \left(\frac{\pi T_{\mathrm{WS}}}{T}\right) \sinh ^{2}\left(\frac{\pi T_{\mathrm{WS}}}{\sqrt{2} T}\right)} .
$$

In deriving this expression, we assumed the following three strong inequalities: $e E \hbar / m_{e}^{2} c^{3} \ll 1, k_{B} T / m_{e} c^{2} \ll 1$, and $e\left(k_{B} T\right)^{2} / E c^{2} \epsilon_{0} \hbar^{2} \ll 1$. Note that these imply the calculation is only valid when the rate of pair production is not too fast. For temperatures much larger than $T_{\mathrm{WS}}$, this equation simplifies to

$$
\begin{aligned}
\Gamma_{S}(E, T) & \approx \frac{m_{e}^{3 / 2} T^{2}\left(k_{B} T\right)^{5 / 2}}{\pi^{9 / 2} \hbar^{4} T_{\mathrm{WS}}^{2}} e^{-\frac{2 m_{e} c^{2}}{k_{B} T}+\frac{\sqrt{e^{3} E / \pi \epsilon_{0}}}{k_{B} T}} \\
& \approx \frac{m_{e}^{5 / 2}\left(k_{B} T\right)^{9 / 2}}{2 \pi^{3} \hbar^{6} \sqrt{\epsilon_{0} e E^{3}}} e^{-\frac{2 m_{e} c^{2}}{k_{B} T}+\frac{\sqrt{e^{3} E / \pi \epsilon_{0}}}{k_{B} T}} .
\end{aligned}
$$

As one can see from this expression, the rate is surprisingly higher for weaker fields. This behavior cannot be extrapolated to arbitrarily weak fields as the validity of our approximations breaks down for $E \lesssim e\left(k_{B} T\right)^{2} / c^{2} \epsilon_{0} \hbar^{2}$.

For a thermal bath of photons in zero electric field, electron-positron pairs can be produced by two-photon fusion (the Breit-Wheeler process). Integrating the cross section for this process over the photon thermal distribution, one finds $[4]^{2}$

$$
\begin{aligned}
\Gamma_{\mathrm{BW}}(T) \approx & 2 \int \frac{d^{3} p}{(2 \pi)^{3}} \frac{d^{3} q}{(2 \pi)^{3}} \frac{p^{\mu} q_{\mu}}{p^{0} q^{0}} \theta\left[\left(p^{\mu}+q^{\mu}\right)^{2}-4 m^{2}\right] \\
& \times f\left(p^{0}\right) f\left(q^{0}\right) \sigma_{\mathrm{BW}}\left[\left(p^{\mu}+q^{\mu}\right)^{2}\right] \\
\approx & \frac{e^{4} m_{e}\left(k_{B} T\right)^{3}}{2(2 \pi)^{4} c^{3} \epsilon_{0}^{2} \hbar^{6}} e^{-\frac{2 m_{e} c^{2}}{k_{B} T}}
\end{aligned}
$$

where $f(E)=1 /\left(e^{E / k_{B} T}-1\right)$ and $\sigma_{\mathrm{BW}}(s)$ is the BreitWheeler cross section [5]. This expression is valid for low temperatures, $k_{B} T / m_{e} c^{2} \ll 1$, up to about $O\left(100 \mathrm{keV} / k_{B}\right)$ with an accuracy of a few percent. In the absence of an intense electric field, higher-order perturbative effects involving more photons are expected to be subdominant if $e k_{B} T / \sqrt{c \hbar \epsilon_{0}} \ll$ $m_{e} c^{2}$ or $k_{B} T \ll 3 m_{e} c^{2}$ [53].

We note that the addition of a constant electric field does not enhance the perturbative Breit-Wheeler process as a constant field consists of zero-energy photons. Beyond this idealized limit, corrections to the Breit-Wheeler rate due to the presence of an additional source of photons with wavelength $\lambda$ are suppressed by $\exp \left[-\lambda m^{2} c^{3} /\left(2 \pi \hbar k_{B} T\right)\right]$. For a typical

\footnotetext{
${ }^{2}$ Note that there are several typos in Ref. [4]. We have repeated the calculation for low temperatures, both fully numerically and in the nonrelativistic approximation, finding agreement with Eq. (8).
}

laser source with $\lambda=0.8 \mu \mathrm{m}$ and a thermal bath of temperature $T=20 \mathrm{keV} / k_{B}$, say, this correction is completely negligible, $\sim 10^{-10^{6}}$.

The Breit-Wheeler process should not be thought of as a competing process to thermal Schwinger pair production. Figure 1(b) shows the dominant Feynman diagram for thermal Schwinger pair production in the intermediate-temperature regime. Applying the Optical Theorem, one can see that a unitarity cut of Fig. 1(b) gives the Breit-Wheeler process [52], except that the effect of the electric field has been accounted for to all orders, giving a nonperturbative enhancement over the pure Breit-Wheeler process. In fact, Eq. (5) reduces to Eq. (8) for temperatures $T \gg T_{\mathrm{CW}}$.

However, in the high-temperature regime, $T>T_{\mathrm{WS}}$, the diagrams of Fig. 1 cease to dominate the rate of pair production and there is a further nonperturbative enhancement that cannot easily be understood diagrammatically. As can be seen from Eqs. (6) and (7), the dependence of the rate, $\Gamma_{S}$, on the fine-structure constant is nonanalytic even after one absorbs one power of $e$ into the electric field.

For completeness, we note that the addition of a single electromagnetic plane wave to a thermal bath of photons also leads to nonperturbatively enhanced electron-positron pair production. This is true even in the long-wavelength limit, $\lambda m c / \hbar \rightarrow \infty$, showing the collective, nonperturbative nature of the phenomenon. In this case, the Breit-Wheeler rate is additively enhanced by [53]

$$
\Gamma_{\text {Plane }} \approx \frac{3^{3 / 4} e^{2}\left(k_{B} T\right)^{2} m^{2}}{16 \pi^{5 / 2} \epsilon_{0} \hbar^{5}}\left(\frac{e E \hbar k_{B} T}{m^{3} c^{5}}\right)^{1 / 4} e^{-\sqrt{\frac{165^{5} m^{3}}{3 e E \hbar k_{B} T}}}
$$

This result is valid for $\sqrt{k_{B} T E /\left(m c^{2} E_{c}\right)} \ll 1$. The crucial difference from that of a constant electric field is that the electromagnetic invariant $E^{2}-c^{2} B^{2}$ of a plane wave vanishes. As we will note later, Eq. (9) is orders of magnitude smaller than the thermal Schwinger rate, showing that the absence of the magnetic field is crucial for pair production.

\section{OBSERVABILITY}

We would like to understand exactly how high the temperatures and how strong the electric fields need to be to get a measurable rate of pair production. To answer that, we will make a simple comparison with the experiment of Ref. [25], which was the first experiment to observe the (multiphoton) Breit-Wheeler process. They observed $106 \pm$ 14 positrons produced in this way, from a total spacetime interaction volume of the order of $10^{-21} \mathrm{~m}^{3} \mathrm{~s}$ (when integrated over all laser shots). Hence we take as our observable reference rate $\Gamma_{\mathrm{Ref}}=10^{23} \mathrm{~m}^{-3} \mathrm{~s}^{-1}=0.1 \mu \mathrm{m}^{-3} \mu \mathrm{s}^{-1}$, which is approximately Avogadro's number of positrons per meter cubed per second. One can therefore reasonably expect that a normalized rate greater than 1 will be required for the rate of pair production to be measurable. In Fig. 2, we show the thermal Schwinger rate in all three regimes, normalized by this reference rate.

The almost perfectly vertical lines of constant rate in the low-temperature regime reflect that in this regime, the thermal enhancements are small. As such, this regime offers no advantages over pure Schwinger pair production for 


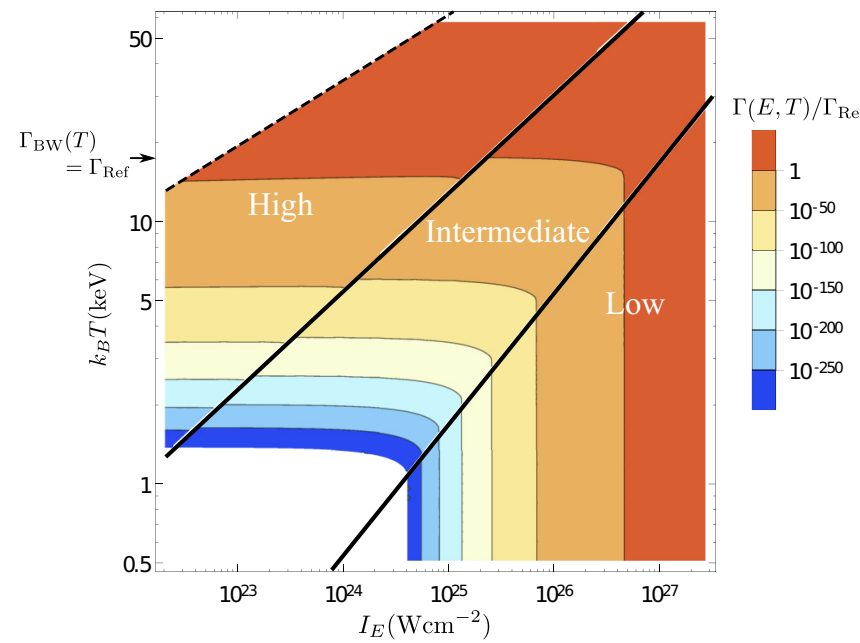

FIG. 2. Approximate rates of pair production for low, medium, and high temperatures. The colored region is where the approximations leading to Eqs. (1), (5), and (6) are well satisfied. All rates are normalized by $\Gamma_{\text {Ref }}=0.1 \mu \mathrm{m}^{-3} \mu \mathrm{s}^{-1}$. For intermediate temperatures, we use only the two leading contributions in the finestructure constant, whereas in the high-temperature regime, all orders are included. This leads to the apparent discontinuity which will be smoothed out by contributions at higher orders.

experimentally observing pair production. On the other hand, in the intermediate- and high-temperature regimes, the thermal enhancements are very significant. Of these two regimes, observing pair production in the high-temperature regime is easier because the electric field intensities required are orders of magnitude smaller, while the temperatures required are very similar.

From Fig. 2, one can see that temperatures around $O\left(20 \mathrm{keV} / k_{B}\right)$ or above are needed in order to produce an observable number of positrons. Perhaps the leading method of producing high-temperature thermal photons is with a laser and cavity, or hohlraum. The aim of achieving inertial confinement fusion (ICF) has been a powerful incentive in developing these technologies. Thermal distributions of $0.3 \mathrm{keV} / k_{B}$ have been achieved since 1990 , though about $0.4 \mathrm{keV} / k_{B}$ is likely the upper limit of this approach [54]. Unfortunately, at these temperatures, the thermal enhancement of the Schwinger rate is negligible.

When ICF is achieved, the burning thermonuclear plasma leads to significantly higher-energy densities. Charged particles in the plasma are expected to reach temperatures from $O\left(20 \mathrm{keV} / k_{B}\right)$ to $O\left(200 \mathrm{keV} / k_{B}\right)$, depending on the composition and size of the burning plasma [55,56]. Burning deuterium (D) plasmas are expected to be hotter than burning deuterium-tritium (DT) plasmas, as the peak nuclear reaction rate is at higher energies for D-D nuclear reactions. For a fixed composition, larger plasmas reach higher temperatures.

As the plasma is not optically thick, the effective temperature of the photons is lower than that of the charged particles. For representative examples of burning deuterium plasma with radii $r=120$ and $r=150 \mu \mathrm{m}$, one finds that the photon-energy density is equal to that of a Planck distribution with two degrees of freedom at $T=22$ and $T=26 \mathrm{keV} / k_{B}$, respectively. The photon distribution can be calculated using

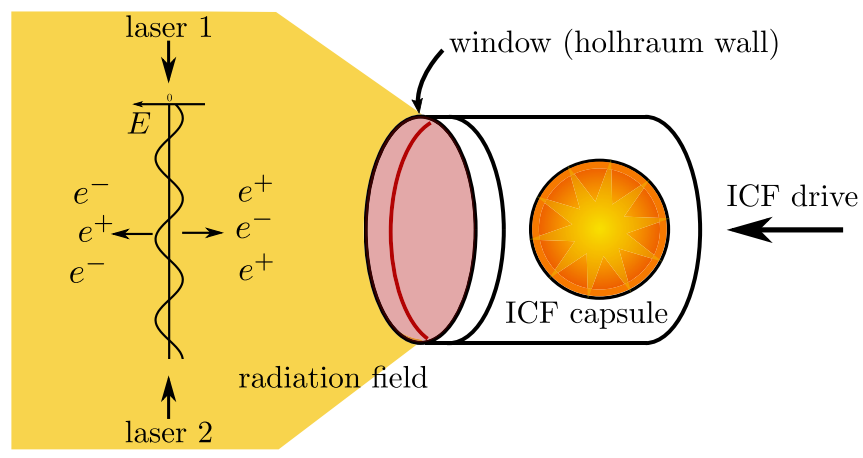

FIG. 3. Schematic of the experimental setup. Two counterpropagating high-energy beams are focused into an $\mathrm{x}$-ray radiation field produced by a burning fusion.

the approach of Ref. [56]. However, the result is further from equilibrium than that of the charged particles. For now, we will assume a thermal distribution of photons, though we will return to this point in Sec. V.

\section{AN EXPERIMENTAL SCHEME}

So, in order to observe the thermal Schwinger process, we would propose combining two lasers with combined intensity $O\left(10^{23} \mathrm{~W} \mathrm{~cm}^{-2}\right)$ with a source of thermal photons with temperature $O\left(20 \mathrm{keV} / k_{B}\right)$. A possible schematic for such an experiment is shown in Fig. 3. The region of interest for our purposes is on the left-hand side, outside the ignited thermonuclear plasma. A window is needed to hold up the material expansion long enough to allow the high-intensity lasers to interact with the radiation from the ICF capsule. The wall of the hohlraum would, in principle, be able to act in this way while transmitting the majority of the radiation, though this would require specific design. As long as the distances from the nuclear plasma are small compared with its radius, the geometric reduction of the intensity will not be significant.

The electric field is provided by a high-intensity laser, split into two counterpropagating beams. These are focused so that the magnetic fields cancel in the vicinity of a given point, whereas the electric fields reinforce. Assuming standard parameters for the laser, with wavelength $\lambda \sim 0.8 \mu \mathrm{m}$, the field maxima of the two beams are expected to be approximately of size $O\left(\lambda^{3}\right)$ and of time extent $O(\lambda / c)$, with approximately 10-20 field maxima per shot, amounting to a possible pairproduction region of size $5 \times 10^{-32} \mathrm{~m}^{3} \mathrm{~s}$. The integrals of the rate over the interaction region can be carried out in the locally constant field approximation (see, for example, Refs. [57,58]), within which the region around the field maxima will dominate the pair production. However, in what follows, we simply multiply the rates by the approximate spacetime volume of the field maxima, which is sufficient to get the order of magnitude correct.

To achieve $\gtrsim 1$ electron-positron pair produced per shot requires a rate $5 \times 10^{6}$ times faster than $\Gamma_{\text {Ref }}$ (see Fig. 4). Assuming a thermal distribution of photons from the burning nuclear plasma, this could be achieved at

$$
\begin{aligned}
T_{\star} & \approx 20 \mathrm{keV} / k_{B}, \\
I_{E \star} & \approx 1.3 \times 10^{23} \mathrm{~W} \mathrm{~cm}^{-2},
\end{aligned}
$$




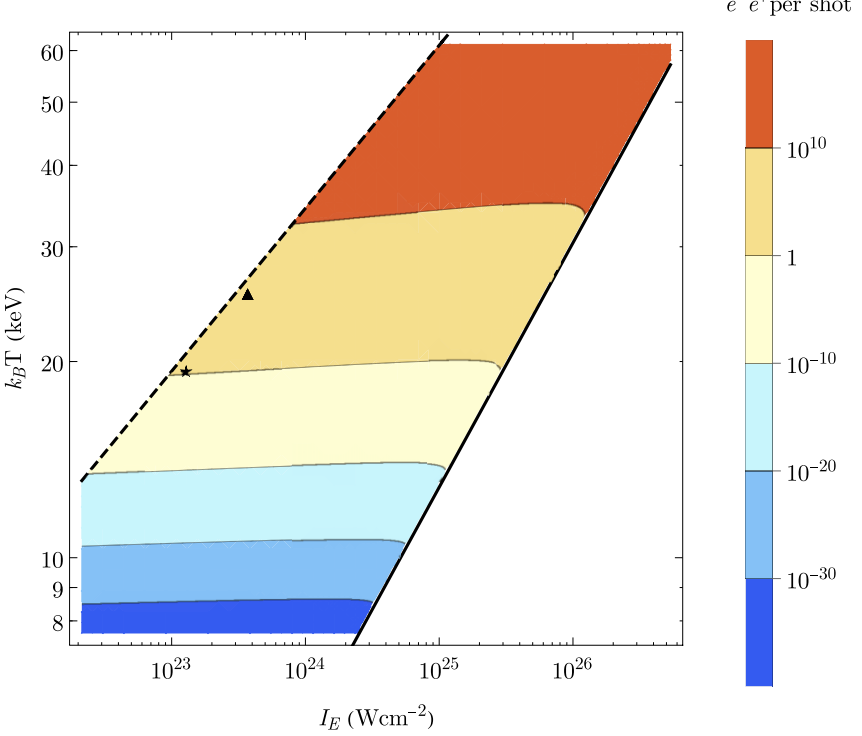

FIG. 4. The number of electron-positron pairs produced per shot in the experiment proposed here. The colored region is the hightemperature region and is where the approximations leading to Eq. (6) are valid. The solid black line is the boundary between the intermediate- and high-temperature regions, defined by $T=T_{\mathrm{Ws}}$. The dashed black line is defined by $E=0.2 e\left(k_{B} T\right)^{2} / \epsilon_{0} c^{2} \hbar^{2}$ and the dotted black line by $T=0.2 m_{e} c^{2} / k_{B}$. The star and diamond are the points referred to in Eqs. (10) and (11).

where $I_{E}$ refers to the combined intensity of the two beams. This parameter point is shown as a star in Fig. 4. For comparison, we also consider a second point with a significantly higher production rate, at

$$
\begin{aligned}
T_{\boldsymbol{\Lambda}} & \approx 26 \mathrm{keV} / k_{B}, \\
I_{E \boldsymbol{\Lambda}} & \approx 3.7 \times 10^{23} \mathrm{~W} \mathrm{~cm}^{-2} .
\end{aligned}
$$

For these two sets of parameters, the numbers of positrons produced per shot via the thermal Schwinger, Breit-Wheeler, and pure Schwinger (without thermal enhancement) processes are given in Table I. We also include the nonperturbative enhancement to the number of positrons produced due to only one of the two laser beams, given by Eq. (9).

Note that the pure Breit-Wheeler process can take place in a larger region than that of the Schwinger pair production, which is not accounted for in Table I. In order to ensure that the thermal Schwinger process dominates, and taking into account its $O\left(10^{6}\right)$ times higher rate, the volume of the interaction region should be significantly less than about

TABLE I. Numbers of positrons produced per shot via different mechanisms for the two sets of parameters given by Eqs. (10) and (11). The column labeled "Eq. (9)" is that for a thermal bath plus a single laser beam (rather than counterpropagating beams).

\begin{tabular}{ccccc}
\hline \hline & Thermal Schwinger & Breit-Wheeler & Eq. (9) & Schwinger \\
\hline$\star$ & 3 & $3 \times 10^{-6}$ & $10^{-167}$ & $10^{-1817}$ \\
$\boldsymbol{\Delta}$ & $1 \times 10^{6}$ & 1 & $10^{-105}$ & $10^{-1062}$ \\
\hline \hline
\end{tabular}

$10^{7} \lambda^{3} \approx 5 \times 10^{-3} \mathrm{~mm}^{3}$. This can be achieved by modifying the diameter of the hohlraum window and by focusing the laser fairly close to the window. Further, the directionality of emitted electrons and positrons can help distinguish between production mechanisms, with the Breit-Wheeler process producing pairs more or less isotropically and the Schwinger process producing pairs along the electric field of the counterpropagating lasers.

It is encouraging that a relatively small increase in both radiation temperature and laser intensity produces such a significant increase in the production rate. One can also see that the thermal Schwinger process has a huge nonperturbative enhancement. A simple perturbative estimate of the number of positrons produced by the Breit-Wheeler process underestimates the actual number by a factor of $10^{6}$. Such large enhancements are a generic feature of the thermal Schwinger process in the high-temperature regime [33].

One can also compare the thermal Schwinger rate to that obtained in a thermal bath plus only a single high-intensity laser beam (in which case the magnetic field does not cancel). In this case, the enhancement of the rate of pair production due to the high intensity laser is given by Eq. (9). For the parameters of either Eqs. (10) or (11), one finds that the enhancement is negligible and the rate of this process is smaller than the thermal Schwinger rate by a factor of $\sim 10^{-100}$ or more, as can be seen in Table I.

For the validity of the locally constant field approximation, it is important that the electric field, as well as the photon distribution from the plasma, are slowly varying on the timescales and length scales of the pair-creation process, described by an instanton. The timescale of the instanton is $t_{\text {inst }} \sim \hbar / k_{B} T$ and the length scale is $\sqrt{e /\left(4 \pi \epsilon_{0} E\right)}[32,33]$. Using temperature and electric field strengths determined by Eq. (10), this amounts to $3 \times 10^{-20} \mathrm{~s} \approx 10^{-11} \mathrm{~m} / \mathrm{c}$ and $5 \times$ $10^{-12} \mathrm{~m}$, respectively. The smallest length scale on which the electric field varies is the wavelength of the laser. Assuming a laser with wavelength of $\lambda \sim 0.8 \mu \mathrm{m}$, one can safely treat the electric field as constant. Further, one would expect the photon distribution from the plasma to vary on a length scale of the order of the size of the hohlraum window. This will likewise be much larger than the length scale of the instanton, $\sim 5 \times$ $10^{-12} \mathrm{~m}$, and hence the locally constant field approximation is applicable.

In the region where the electric field and thermal photons collide, electrons and positrons will be produced with an approximately thermal spectrum of velocities and then accelerated in opposite directions antiparallel and parallel, respectively, to the electric field. Their thermal velocities are isotropic in the laboratory frame and are expected to be rather large, $\frac{1}{3} \bar{v}^{2} \sim k_{B} T / m_{e} \sim(0.2 c)^{2}$. The field then accelerates the particles over a distance $\lesssim \lambda$, giving them a highly relativistic velocity, $v \approx c$, parallel to the electric field and up to energies of the order of $e E \lambda \sim 1 \mathrm{GeV}$. Once produced, the electrons and positrons may be deflected in opposite directions with a magnet, after which their momenta can be measured by a calorimeter, as in Ref. [25]. If the combined intensity of the lasers is greater than around $10^{24} \mathrm{~W} \mathrm{~cm}^{-1}$, a seed electronpositron pair produced by the thermal Schwinger process will induce a cascade of pair production, so amplifying any positive signal [22-24]. 


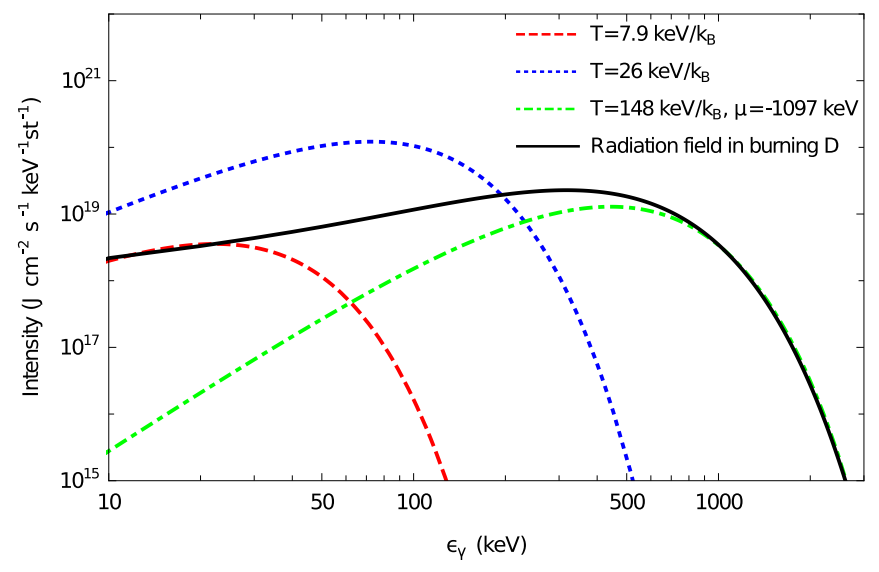

FIG. 5. Photon intensity in a D-burning target of radius $150 \mu \mathrm{m}$, along with various approximations to it. Note that a purely thermal distribution at $T=148 \mathrm{keV} / k_{B}$ would lie at much higher intensities.

In the absence of charged particles, the thermal Schwinger process is the dominant mechanism of electron-positron pair production. However, if charged particles are not adequately shielded, other pair-production processes are possible, such as the trident mechanism $\left(e^{-} Z \rightarrow e^{-} e^{+} e^{-} Z\right)$ and the BetheHeitler process $\left(\gamma Z \rightarrow e^{+} e^{-} Z\right)$. Another possibility is for nonlinear Compton scattering of charged particles in the laser field, producing high-energy photons which then take part in the Breit-Wheeler process. Debye screening by charged particles will also inhibit the thermal Schwinger process if the Debye length is not much longer than the length scale of the pair-creation process, $\sqrt{e /\left(4 \pi \epsilon_{0} E\right)}$. For the parameters of Eq. (10), one requires the density of charged particles to be much less than one per $\mathrm{pm}^{3}$. In the regime we have considered here, the purely thermal and the purely Schwinger pair-production rates are orders of magnitude lower than the combination. Thus, by performing null shots, with either only the burning plasma or only the high-intensity laser, one can measure the presence of any backgrounds.

\section{PHOTON DISTRIBUTIONS}

Let us return to consider the distribution of photons in the burning plasma. This must be close to equilibrium for our approach to be valid. To investigate this, we have solved the Boltzmann equation for the distribution of photons for a range of different plasma sizes and compositions. We have followed the method of Ref. [56], including the effect of Compton scattering. For our representative example of a burning deuterium plasma of radius $r=150 \mu \mathrm{m}$, the photon intensity at the surface of this plasma is shown as the full black line in Fig. 5.

Equating the photon-energy density to that of a thermal distribution, one finds that the effective temperature of the distribution is $26 \mathrm{keV} / k_{B}$. Doing the same for the photonnumber density, one instead finds a somewhat lower effective temperature of $16 \mathrm{keV} / k_{B}$, showing that the distribution is shifted to higher energies with respect to a thermal distribution. Plotting the photon intensity of a Planck distribution at $T=26 \mathrm{keV} / k_{B}$, i.e., the blue dotted line in Fig. 5, one can see the shift to higher energies.
The high-energy tail of the distribution, above about $700 \mathrm{keV}$, is an exponential fall off and hence fits well with a Boltzmann tail with an effective temperature of $T=$ $148 \mathrm{keV} / k_{B}$, though scaled down by a normalization or, equivalently, a negative photon chemical potential ${ }^{3}, \mu=$ $-1097 \mathrm{keV}$, plotted as the dot-dashed green line in Fig. 5. At the lowest energies, the distribution rises above this and can be better described by a purely thermal distribution at a much lower temperature, $T=7.9 \mathrm{keV} / k_{B}$, plotted as the dashed red line in Fig. 5. At intermediate energies, the distribution is not well described by a Bose-Einstein distribution. Nevertheless, the overall shape of the distribution is qualitatively similar to a thermal distribution, being smooth and highly occupied with a powerlike rise at low energies and an exponential decrease at high energies, though we have used four different effective temperatures to describe different aspects of it, ranging from 7.9 to $148 \mathrm{keV} / k_{B}$.

In two counterpropagating laser beams with intensity given by Eq. (10) or (11), one finds that the intermediatetemperature regime of thermal Schwinger pair production would be reached at temperatures above

$$
\begin{aligned}
T_{\mathrm{CW}, \star} & =0.20 \mathrm{keV} / k_{B}, \\
T_{\mathrm{CW}, \boldsymbol{}} & =0.32 \mathrm{keV} / k_{B},
\end{aligned}
$$

and the high-temperature regime would be reached at temperatures above

$$
\begin{aligned}
T_{\mathrm{WS}, \star} & =2.5 \mathrm{keV} / k_{B}, \\
T_{\mathrm{WS}, \boldsymbol{\Delta}} & =3.7 \mathrm{keV} / k_{B} .
\end{aligned}
$$

All four effective temperatures that we have used to describe the distribution of photons in the burning plasma are well above these temperatures. We thus expect the hightemperature regime to provide a better description of pair production in this setup than either the low- or intermediatetemperature regimes, which would imply that the diagrams of Fig. 1 do not dominate pair production and there is a nonperturbative enhancement over both pure Schwinger and pure thermal pair production.

Physically, it is clear that the process of pair production should not depend on the photon gas being precisely in equilibrium: if we use the picture of tunneling from an excited state, one would expect that it is the energy and density of the photon distribution, rather than the nearness to equilibrium, that matters.

On the other hand, the condition of equilibrium is necessary for the calculation because it leads to important simplifications in the calculation of the production rate. The nonperturbative calculation of Eq. (6) [32,33] relied heavily on the Matsubara formalism $[59,60]$, which is only valid in equilibrium. In the high-temperature regime, a resummation of all orders of the perturbative loop expansion was required. Generalizing the result to any out-of-equilibrium distribution is beyond the scope of this paper.

\footnotetext{
${ }^{3}$ The photon chemical potential must be zero in equilibrium, but not necessarily out of equilibrium. In this context its presence is natural as photon number conserving processes dominate.
} 
We note, however, that the diagrams of Fig. 1 can be calculated in an arbitrary photon distribution, following the approach of Ref. [52], though in the high-temperature regime these diagrams are not dominant. Considering the calculation in this distribution, it can be seen that these diagrams reproduce the perturbative Breit-Wheeler rate up to very small corrections, essentially because the photon gas is highly occupied at energies much greater than $k_{B} T_{\mathrm{CW}}$ [see Eqs. (2) and (12)]. Further, perturbative corrections in this distribution due to the high-intensity laser require one photon from the highenergy tail of the distribution and hence are suppressed by $\exp \left(-\lambda m^{2} c^{3} /\left(2 \pi \hbar k_{B} T\right)+\mu / k_{B} T\right) \sim 10^{-10^{5}}$, where $T$ and $\mu$ refer to the green dot-dashed line in Fig. 5. Thus any enhancement due to the high-intensity laser must be a nonperturbative phenomenon which goes beyond the diagrams of Fig. 1.

Because the full nonperturbative calculation of the rate of pair production is beyond the scope of this paper, the possibility of nonperturbative enhancements in our proposed setup is conjectural. However, as all the effective temperatures we have used to describe the photon distribution are larger than $T_{\mathrm{WS}}$ [Eq. (13)], we expect the high-temperature regime to best describe the photon distribution in question.
We thus expect a nonperturbative enhancement over the perturbative prediction, as is the case in equilibrium where the enhancement to the positron yield was $O\left(10^{6}\right)$. The experiment we have proposed here would be able to test this plausible conjecture by performing null shots without the counterpropagating laser beams, for which only the perturbative process is possible. This would be able to determine which features of a photon distribution are important for the nonperturbative enhancements to pair production which feature in the thermal Schwinger effect and how generic such enhancements are.

\section{ACKNOWLEDGMENTS}

O.G. would like to thank Holger Gies and Greger Torgrimsson for discussions related to this work. O.G. was supported by the Research Funds of the University of Helsinki. S.M. was supported by Engineering and Physical Sciences Research Council Grant No. EP/M018555/1 and by Horizon 2020 under European Research Council Grant Agreement No. 682399. A.R. was supported by the UK Science and Technology Facilities Council Grant No. ST/P000762/1.
[1] J. S. Schwinger, On gauge invariance and vacuum polarization, Phys. Rev. 82, 664 (1951).

[2] C. Danson, D. Hillier, N. Hopps, and D. Neely, Petawatt class lasers worldwide, High Power Laser Sci. Eng. 3, e3 (2015).

[3] I. C. E. Turcu et al., High field physics and QED experiments at ELI-NP, Rom. Rep. Phys. 68, S145 (2016).

[4] T. A. Weaver, Reaction rates in a relativistic plasma, Phys. Rev. A 13, 1563 (1976).

[5] G. Breit and J. A. Wheeler, Collision of two light quanta, Phys. Rev. 46, 1087 (1934).

[6] O. J. Pike, F. Mackenroth, E. G. Hill, and S. J. Rose, A photonphoton collider in a vacuum hohlraum, Nat. Photon. 8, 434 (2014).

[7] R. Ruffini, G. Vereshchagin, and S.-S. Xue, Electron-positron pairs in physics and astrophysics: From heavy nuclei to black holes, Phys. Rep. 487, 1 (2010).

[8] E. Brezin and C. Itzykson, Pair production in vacuum by an alternating field, Phys. Rev. D 2, 1191 (1970).

[9] A. Ringwald, Pair production from vacuum at the focus of an x-ray free electron laser, Phys. Lett. B 510, 107 (2001).

[10] V. S. Popov, Schwinger mechanism of electron positron pair production by the field of optical and x-ray lasers in vacuum, Pisma Zh. Eksp. Teor. Fiz. 74, 151 (2001) [JETP Lett. 74, 133 (2001)].

[11] A. Di Piazza, Pair production at the focus of two equal and oppositely directed laser beams: The effect of the pulse shape, Phys. Rev. D 70, 053013 (2004).

[12] G. V. Dunne, H. Gies, and R. Schutzhold, Catalysis of Schwinger vacuum pair production, Phys. Rev. D 80, 111301(R) (2009).

[13] A. Otto, D. Seipt, D. Blaschke, S. A. Smolyansky, and B. Kämpfer, Dynamical Schwinger process in a bifrequent electric field of finite duration: survey on amplification, Phys. Rev. D 91, 105018 (2015).
[14] A. D. Panferov, S. A. Smolyansky, A. Otto, B. Kämpfer, D. B. Blaschke, and A. Juchnowski, Assisted dynamical Schwinger effect: Pair production in a pulsed bifrequent field, Eur. Phys. J. D 70, 56 (2016).

[15] G. Torgrimsson, J. Oertel, and R. Schützhold, Doubly assisted Sauter-Schwinger effect, Phys. Rev. D 94, 065035 (2016).

[16] C. Kohlfurst, M. Mitter, G. von Winckel, F. Hebenstreit, and R. Alkofer, Optimizing the pulse shape for Schwinger pair production, Phys. Rev. D 88, 045028 (2013).

[17] M. J. A. Jansen and C. Müller, Strongly enhanced pair production in combined high- and low-frequency laser fields, Phys. Rev. A 88, 052125 (2013).

[18] C. Muller, A. B. Voitkiv, and N. Grun, Differential rates for multiphoton pair production by an ultrarelativistic nucleus colliding with an intense laser beam, Phys. Rev. A 67, 063407 (2003).

[19] P. Sieczka, K. Krajewska, J. Z. Kamiński, P. Panek, and F. Ehlotzky, Electron-positron pair creation by powerful laser-ion impact, Phys. Rev. A 73, 053409 (2006).

[20] A. Di Piazza, E. Lotstedt, A. I. Milstein, and C. H. Keitel, Barrier Control in Tunneling $e^{+}-e^{-}$Photoproduction, Phys. Rev. Lett. 103, 170403 (2009).

[21] F. Fillion-Gourdeau, E. Lorin, and A. D. Bandrauk, Resonantly Enhanced Pair Production in a Simple Diatomic Model, Phys. Rev. Lett. 110, 013002 (2013).

[22] A. R. Bell and J. G. Kirk, Possibility of Prolific Pair Production with High-Power Lasers, Phys. Rev. Lett. 101, 200403 (2008).

[23] A. M. Fedotov, N. B. Narozhny, G. Mourou, and G. Korn, Limitations on the Attainable Intensity of High Power Lasers, Phys. Rev. Lett. 105, 080402 (2010).

[24] E. N. Nerush, I. Yu. Kostyukov, A. M. Fedotov, N. B. Narozhny, N. V. Elkina, and H. Ruhl, Laser Field Absorption in SelfGenerated Electron-Positron Pair Plasma, Phys. Rev. Lett. 106, 035001 (2011) [Erratum: 106, 109902 (2011)]. 
[25] D. L. Burke, R. C. Field, G. Horton-Smith, J. E. Spencer, D. Walz, S. C. Berridge, W. M. Bugg, K. Shmakov, A. W. Weidemann, C. Bula et al., Positron Production in Multiphoton Light by Light Scattering, Phys. Rev. Lett. 79, 1626 (1997).

[26] F Mackenroth and A. Di Piazza, Nonlinear compton scattering in ultrashort laser pulses, Phys. Rev. A 83, 032106 (2011).

[27] A. G. R. Thomas, C. P. Ridgers, S. S. Bulanov, B. J. Griffin, and S. P. D. Mangles, Strong Radiation-Damping Effects in a Gamma-Ray Source Generated by the Interaction of a HighIntensity Laser with a Wakefield-Accelerated Electron Beam, Phys. Rev. X 2, 041004 (2012).

[28] T. G. Blackburn, C. P. Ridgers, J. G. Kirk, and A. R. Bell, Quantum Radiation Reaction in Laser-Electron-Beam Collisions, Phys. Rev. Lett. 112, 015001 (2014).

[29] J. M. Cole, K. T. Behm, E. Gerstmayr, T. G. Blackburn, J. C. Wood, C. D. Baird, M. J. Duff, C. Harvey, A. Ilderton, A. S. Joglekar et al., Experimental Evidence of Radiation Reaction in the Collision of a High-Intensity Laser Pulse with a LaserWakefield Accelerated Electron Beam, Phys. Rev. X 8, 011020 (2018).

[30] K. Poder, M. Tamburini, G. Sarri, A. Di Piazza, S. Kuschel, C. D. Baird, K. Behm, S. Bohlen, J. M. Cole, D. J. Corvan et al., Experimental Signatures of the Quantum Nature of Radiation Reaction in the Field of an Ultraintense Laser, Phys. Rev. X 8, 031004 (2018).

[31] A. Di Piazza, C. Muller, K. Z. Hatsagortsyan, and C. H. Keitel, Extremely high-intensity laser interactions with fundamental quantum systems, Rev. Mod. Phys. 84, 1177 (2012).

[32] O. Gould and A. Rajantie, Thermal Schwinger pair production at arbitrary coupling, Phys. Rev. D 96, 076002 (2017).

[33] O. Gould, A. Rajantie, and C. Xie, Worldline sphaleron for thermal Schwinger pair production, Phys. Rev. D 98, 056022 (2018).

[34] P. Cvitanovic, Asymptotic estimates and gauge invariance, Nucl. Phys. B 127, 176 (1977).

[35] I. K. Affleck, O. Alvarez, and N. S. Manton, Pair production at strong coupling in weak external fields, Nucl. Phys. B 197, 509 (1982).

[36] S. L. Lebedev and V. I. Ritus, Virial representation of the imaginary part of the Lagrange function of an electromagnetic field, Zh. Eksp. Teor. Fiz. 86, 408 (1984) [Sov. Phys. JETP 59, 237 (1984)].

[37] I. Huet, M. Rausch de Traubenberg, and C. Schubert, Asymptotic behavior of the QED perturbation series, Adv. High Energy Phys. 2017, 6214341 (2017).

[38] B. Acharya et al. (MoEDAL), Search for Magnetic Monopoles with the Moedal Forward Trapping Detector in $13 \mathrm{TeV}$ ProtonProton Collisions at the LHC, Phys. Rev. Lett. 118, 061801 (2017).

[39] O. Gould and A. Rajantie, Magnetic Monopole Mass Bounds from Heavy Ion Collisions and Neutron Stars, Phys. Rev. Lett. 119, 241601 (2017).

[40] V. I. Ritus, The Lagrange function of an intensive electromagnetic field and quantum electrodynamics at small distances,
Pisma Zh. Eksp. Teor. Fiz. 69, 1517 (1975) [Sov. Phys. JETP 42, 774 (1975)].

[41] V. I. Ritus, Connection between strong-field quantum electrodynamics with short-distance quantum electrodynamics, Zh. Eksp. Teor. Fiz. 73, 807 (1977) [JETP 46, 423 (1977)].

[42] V. I. Ritus, Effective Lagrange function of intense electromagnetic field in QED, in Frontier Tests of QED and Physics of the Vacuum, edited by E. Zavattini, D. Bakalov, and C. Rizzo, Vol. 1 (Sofia, Hungary, 1998), p. 11.

[43] H. Gies and F. Karbstein, An Addendum to the HeisenbergEuler effective action beyond one loop, J. High Energ. Phys. 03 (2017) 108.

[44] H. Gies, QED effective action at finite temperature: Two-loop dominance, Phys. Rev. D 61, 085021 (2000).

[45] A. Monin and M. B. Voloshin, Photon-stimulated production of electron-positron pairs in an electric field, Phys. Rev. D 81, 025001 (2010).

[46] K. G. Selivanov, Tunneling at finite temperature, Phys. Lett. A 121, 111 (1987).

[47] B. I. Ivlev and V. I. Mel'nikov, Tunneling and activated motion of a string across a potential barrier, Phys. Rev. B 36, 6889 (1987).

[48] A. R. Brown, Schwinger pair production at nonzero temperatures or in compact directions, Phys. Rev. D 98, 036008 (2018).

[49] P. Draper, Virtual and thermal Schwinger processes, Phys. Rev. D 98, 125014 (2018).

[50] L. Medina and M. C. Ogilvie, Schwinger pair production at finite temperature, Phys. Rev. D 95, 056006 (2017).

[51] M. Korwar and A. M. Thalapillil, Finite temperature Schwinger pair production in coexistent electric and magnetic fields, Phys. Rev. D 98, 076016 (2018).

[52] G. Torgrimsson, Thermally versus dynamically assisted Schwinger pair production, Phys. Rev. D 99, 096007 (2019).

[53] B. King, H. Gies, and A. Di Piazza, Pair production in a plane wave by thermal background photons, Phys. Rev. D 86, 125007 (2012); 87, 069905(E) (2013).

[54] J. D. Lindl, P. Amendt, R. L. Berger, S. G. Glendinning, S. H. Glenzer, S. W. Haan, R. L. Kauffman, O. L. Landen, and L. J. Suter, The physics basis for ignition using indirect-drive targets on the National Ignition Facility, Phys. Plasmas 11, 339 (2004).

[55] M. Tabak, What is the role of tritium-poor fuels in ICF? Nucl. Fusion 36, 147 (1996).

[56] S. J. Rose, Electron-positron pair creation in burning thermonuclear plasmas, High Energy Density Phys. 9, 480 (2013).

[57] D. V. Gal'tsov and N. S. Nikitina, Macroscopic vacuum effects in an inhomogeneous and nonstationary electromagnetic field, Zh. Eksp. Teor. Fiz. 85, 1217 (1983) [Sov. Phys. JETP 57, 705 (1983)].

[58] S. P. Gavrilov and D. M. Gitman, Vacuum instability in slowly varying electric fields, Phys. Rev. D 95, 076013 (2017).

[59] F. Bloch, Zur theorie des austauschproblems und der remanenzerscheinung der ferromagnetika, Z. Physik 74, 295 (1932).

[60] T. Matsubara, A new approach to quantum statistical mechanics, Prog. Theor. Phys. 14, 351 (1955). 